\title{
Comparative Modeling of Gingival Protein and Docking Studies with Natural Flavonoid Inhibitors
}

\author{
ABHIGNA $\mathrm{P}^{1}$, JAYASREE $\mathrm{D}^{2}$ and SHRAVAN KUMAR GUNDA ${ }^{1}$ \\ ${ }^{1}$ Bioinformatics Division, Osmania University, Hyderabad, Telangana, India \\ ${ }^{2}$ University College of Science, Saifabad, Osmania University, Hyderabad, Telangana, India \\ gunda14@gmail.com
}

Received 12 February 2016 / Accepted 26 February 2016

\begin{abstract}
The study of protein structure information provides fundamental aspects into most biochemical functions and consequently into the cause of diseases and possible treatment. Most of the protein structures were not solved experimentally, for this it requires x-ray crystallography, NMR and electron microscopy. Hence, in silico protein structure prediction methods are useful to generate a protein structure. Molecular modeling of proteins are rapidly growing field. The present study deals with homology modeling of B4DJI1 ( $L$-lactate dehydrogenase) protein of gingival crevicular fluid (Gingivitis) by using Modeller 9.15. The model shows that $91.1 \%$ of amino acid residues in most favored region. The predicted model was then used to perform molecular docking simulations with natural flavonoid derivatives to assess their ability. The ligand binding residueswere found to be similar to the predicted active site residues.
\end{abstract}

Keywords: Homology modeling, Periodontal disease, Gingivalcrevicular fluid, Autodock

\section{Introduction}

Periodontal diseases are most common in humans ${ }^{1}$. They are activated by bacterial biofilms that complywith the tooth surfaces and cause a local inflammatory response in the gingival tissues. Under the influence of environmental factors, genetic tendencies; gingivitis may progress ${ }^{2,3}$. Periodontal infectionmay also contribute to side effects including atherosclerosis and diabetes mellitus. Gingival cervicular fluidis considered as a suitable, easily accessible alternative source of biological material that can be used in the study of gingival inflammation ${ }^{4}$.

Flavonoids are poly-phenolic compounds distributed in vegetables and fruits and have been suggested to be most bioactive compounds in the diet that are responsible for decreasing the risk of cancer. These are plant secondary metabolites and have low molecular weight, which are widespread in the plants and foods of plant origin ${ }^{5}$. Polyphenols and phenols are the most potent chemo-protective agents. Plant flavonoid derivatives are of outstanding importance and contain a wide spread of polyphenols that include flavonols (myricetin, quercetin) flavones, chalcones, flavanidiols, anthocyanins, anthocyanidins $^{6}$. These nontoxic ingredients, found in several foods have been demonstrated 
to possess protective properties. For instance, flavonoid derivatives have been reported to have multiple biological activities including anti-inflammatory, anti-fungal, anti-cancer, anti-viral, anti-bacterial and anti-oxidant activities ${ }^{7}$. Considering the increasing interest in anticancer activities of natural phenolic compounds especially flavonoids, molecular modeling and docking studies were accomplished in this work with the object to find mathematical models that could aid in understanding and be used for prediction of the anticancer activity of flavonoids.

In this study, MODELLER9.15 was used to generate 3D model of Putative high mobility group protein B1-like 1 (uniprot accession number: B4DJI1) protein from human. Human Heart $L$-Lactate Dehydrogenase $H$ Chain, Ternary complex with NADH and oxamate (PDB id: 1IOZ:A), is used as template for model build up. Validation of model was done by PROCHECK program. Active site prediction was performed by using 3D ligand site, an online active site prediction tool and molecular docking study was performed using AutoDock4.2.

\section{Experimental}

\section{Sequence alignment and structure prediction}

The FASTA sequence of query protein (B2RPK0) was retrieved from uniprot KB protein sequence database search (http://www.uniprot.org/uniprot/B4DJI1). Following BLASTp run using NCBI protein BLAST (http://blast.ncbi.nlm.nih.gov/Blast.cgi), "Human Heart $L$-Lactate Dehydrogenase H Chain, Ternary complex with NADH and oxamate (PDB id: 1I0Z:A) was selected as template. The selected template was downloaded from RCSB, a primary protein structure database (http://www.rcsb.org/pdb). Both the sequences were subjected to pairwise alignment using ClustalX. The 3D-structure of query protein was predicted by automated homology modeling program, Modeller9.15 ${ }^{8}$. The template and query sequences were aligned carefully to remove potential alignment errors. Loop building was performed by using SPDB viewer' ${ }^{9}$. The final validation of the model was done by Ramachandran plot analysis.

MODELLER 9.15 was then used to gain satisfactory models; an automated approach to comparative modeling by satisfaction of spatial restrains ${ }^{10}$. After manually modifying the alignment input file in MODELLER 9.15 to match the template and query sequence, 20 models were generated and were then minimized using the molecular dynamics and simulation procedure CHARMM in MODELLER for each of the primary sequences out of which the models with least modeller objective function were then chosen. These models were then checked in detail for the protein structure stereochemistry by using PROCHECK $^{11}$, which generates Ramachandran plots and comprehensive residue by residue listing facilitates, the in depth assessment of Psi/Phi angles and the backbone conformation of the models.

\section{Docking studies}

Molecular docking studies were carried out using Autodock 4.2. The initial flavonoid structures were sketched in SYBYL 6.7 and minimized using Gasteiger-Huckel charges by Powell gradient method and convergence criterion was selected as $0.005 \mathrm{kcal} / \mathrm{mol}^{12}$. These molecules were saved in $\mathrm{MO}^{12}$ format. Molecular docking study was carried out using Autodock 4.2 software $^{13}$, which uses Genetic algorithm (GA). DNA Topoisomerase II protein was loaded into AutoDock Tools (ADT) (http://autodock.scripps.edu/ resources) as a receptor and made ready for docking. After loading the protein, hydrogens were added using 
the edit option in ADT. Initially, the odeled PDB file does not contain any hydrogens. The ligands were separately and individually docked with odeled B4DJI1 protein, a grid for dock search was built for the molecule to find the most probable binding site of B4DJI1 and to measure its interaction parameters with synthesized molecules. The docking process was carried out in the default parameters of ADT. Active sites have been predicted by submitting the predicted homology model to 3Dligand site, a binding site prediction server (http://www.sbg.bio.ic.ac.uk/3dligandsite/advanced.cgi) $)^{14}$.

\section{Results and Discussion}

\section{Structural evaluation}

This study reports that the template protein (PDB ID: 1IOZ) having high degree of homology with Gingivitis protein was used as a template with good atomic resolution of its crystal structure. The secondary structure alignment obtained between the query and template sequence is shown in Figure 1. The query (B4DJI1) subjected for homology modeling by Modeller ended up with a loop by default modeling process. The $G$-factors indicating the quality of covalent and bond angle distance were The predicted model was subjected to PROCHECK analysis to determine psi and phi torsion angles, the comparable Ramachandran plot characteristics and $G$-factors confirm the quality of predicted model. The PROCHECK software is used for validation of odeled protein. It generates a number of files which list complete residue by residue data and the assessment of the overall quality of the generated structure as compared to well refined structures of the same resolution. The Ramachandran plot of gingivitis shows 245 amino acid residues $(91.1 \%)$ in most favorable region with 22 amino acid residues $(8.2 \%)$ falling in to additionally allowed regions, twoamino acids $(0.7 \%)$ falling in to generously allowed region and there is zero amino acids falling in disallowed region. These results clearly indicate that the generated models are much more sophisticated and more conformationally better. Figure 2 for Ramachandran plot of gingivitis protein B4DJI1.

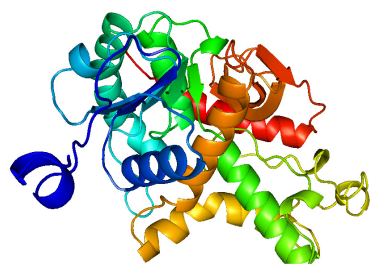

Figure 1. Secondary structure of predicted B4DJI1protein

\section{Docking results}

The study of 5 natural flavonoid compounds has revealed that the amino residues can frequently interact with B4DJI1 binding site and that these residues are responsible for the selectivity of flavonoid inhibitors.

The docked poses of all the compounds with B4DJI1 are shown in Figure 3 which clearly demonstrated the binding positions of the ligand with the protein. Analysis of the receptor/ligand complex models generated after successful molecular docking of the natural flavonoids quercetin, naringenin, hesperetin and leucocyanidin, kaempferol, based on the parameters such as, hydrogen bonds distance, amino acid interactions, binding energy and orientation of the docked compounds within the active site. As a general rule, both hydrogen bond and hydrophobic interactions between the compound and the active sites of the receptor have been found to be responsible for mediating the biological activity. 


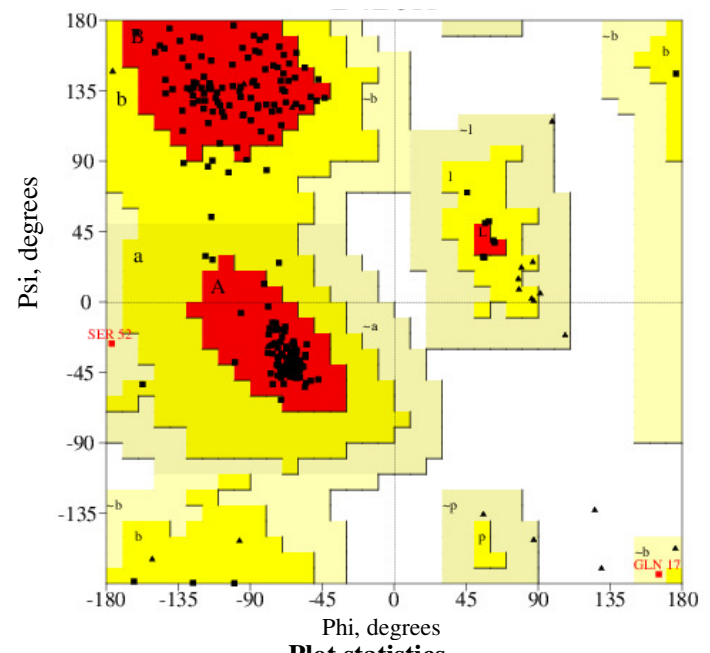

Plot statistics

Residues in most favoured regions [A,B,J]

Residues in additional allowed regions $[\mathrm{a}, \mathrm{b}, 1, \mathrm{p}]$

Residues in generously allowed regions $[-a,-b,-1,-p]$

Residues in disallowed regions

Number of non-glycine and non-proline residue

Number of end-residues (excl. Gly and Pro)

Number of glycine residue (Shown as triangles)

Number of proline residues

Total number of residues

\begin{tabular}{ll}
0 & $0 \%$ \\
\hline 269 & $100 \%$
\end{tabular}

Figure 2. Ramachadran plot of predicted B4DJI1 protein

As shown in Table 1, hesperetin showed highest binding energy $-6.74 \mathrm{kcal} / \mathrm{mol}$ when compared to quercetin, naringenin, leucocyanidin, and kaempferol(-6.49, -5.84, -6.38, 6.05 and $-8.58 \mathrm{kcal} / \mathrm{mol}$ respectively). Moreover, in naringenin having five interactions followed by other three ligandsquercetin, naringenin, leucocyanidin, which are having four interactions each. Kaempferol shows only two interactions. Naringenin shows highest number of interactions i.e., 5 interactions. This proves that almost all flavonoids show good interactions with modelled B4DJI1 protein binding site.

Table 1. Molecular docking interactions and their binding energy

\begin{tabular}{|c|c|c|c|c|}
\hline $\begin{array}{c}\text { Name of the } \\
\text { Ligand }\end{array}$ & Interacting amino acids & $\begin{array}{l}\text { Grid X-Y-Z } \\
\text { coordinates }\end{array}$ & $\begin{array}{c}\text { Binding } \\
\text { energy } \Delta \mathrm{G} \text {, } \\
\text { Kcal/Mol }\end{array}$ & $\begin{array}{c}\text { Dissociatio } \\
\mathrm{n} \text { constant } \\
(\mathrm{kl}), \mu \mathrm{M}\end{array}$ \\
\hline Quercetin & $\begin{array}{c}\text { Val28, Ala71, Asn111, } \\
\text { Val109 }\end{array}$ & $\begin{array}{c}10.711 \\
40.405,50.267\end{array}$ & -6.49 & 17.53 \\
\hline Naringenin & $\begin{array}{l}\text { Ala31, Arg72, Asn86, } \\
\text { Asn111, Ala71 }\end{array}$ & $\begin{array}{c}10.711 \\
40.405,50.267\end{array}$ & -5.84 & 52.45 \\
\hline Kaempferol & $\operatorname{Arg} 142$, Ser110 & $\begin{array}{c}10.711 \\
40.405,50.267\end{array}$ & -6.05 & 36.64 \\
\hline Leucocyanidin & $\begin{array}{c}\text { Asn111, ARg142, Ala71, } \\
\text { Ser134 }\end{array}$ & $\begin{array}{c}10.711 \\
40.405,50.267\end{array}$ & -6.38 & 20.98 \\
\hline Hesperetin & $\begin{array}{c}\text { Asn111, Thr221, Arg72, } \\
\text { Val31 }\end{array}$ & $\begin{array}{c}10.711, \\
40.405,50.267\end{array}$ & -6.74 & 11.55 \\
\hline
\end{tabular}



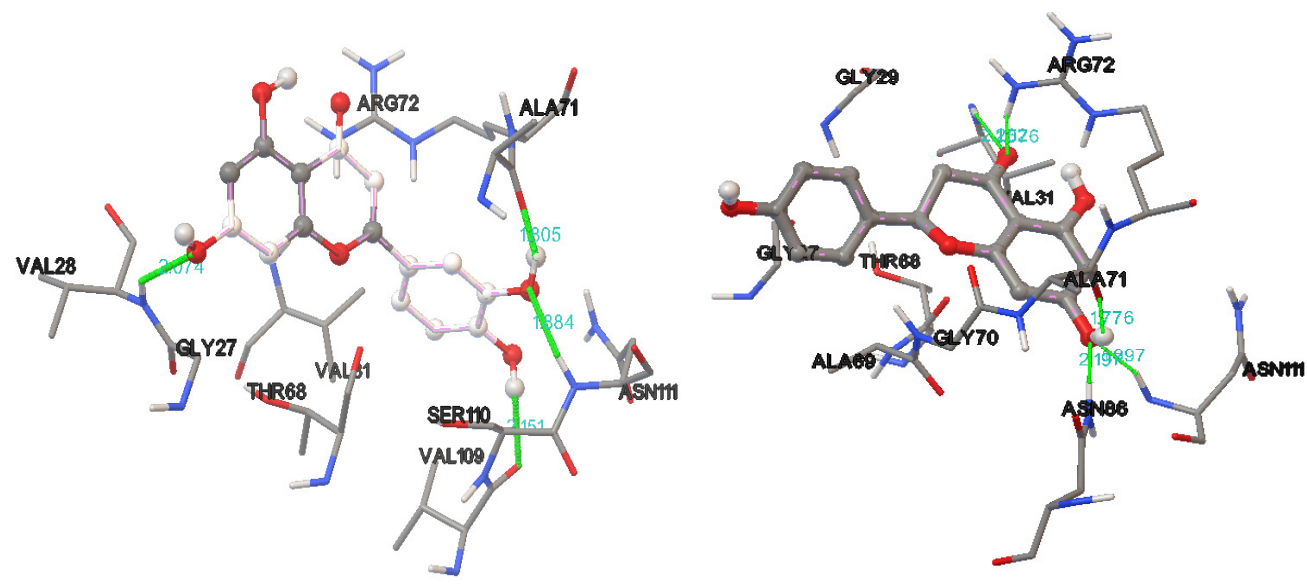

Quercetin

Naringenin

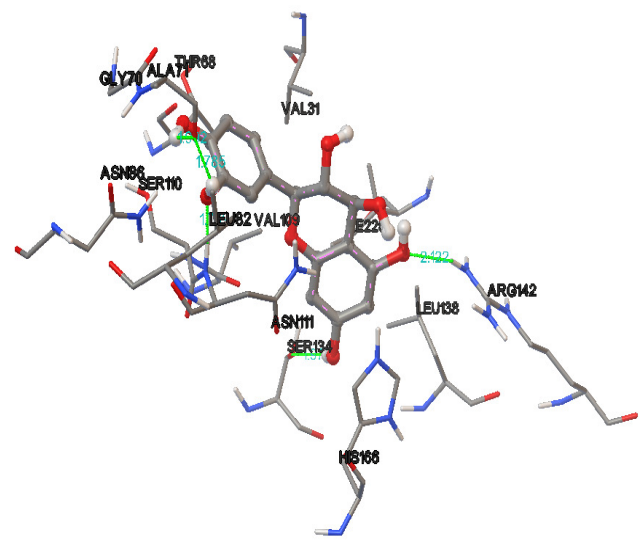

Leucocyanidin

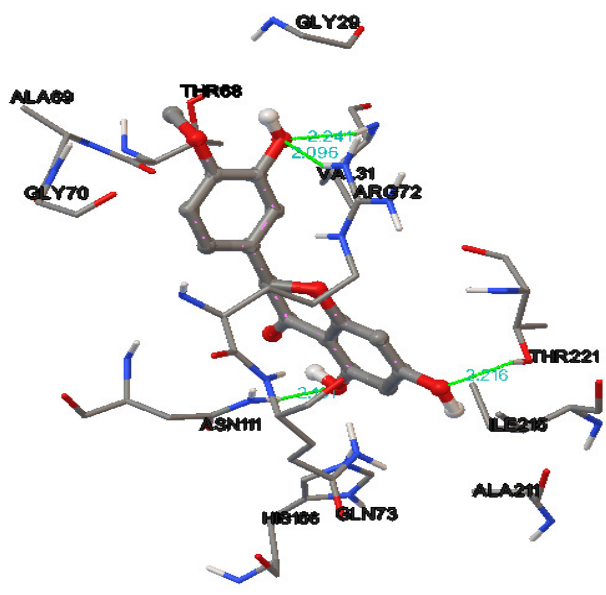

Hesperetin

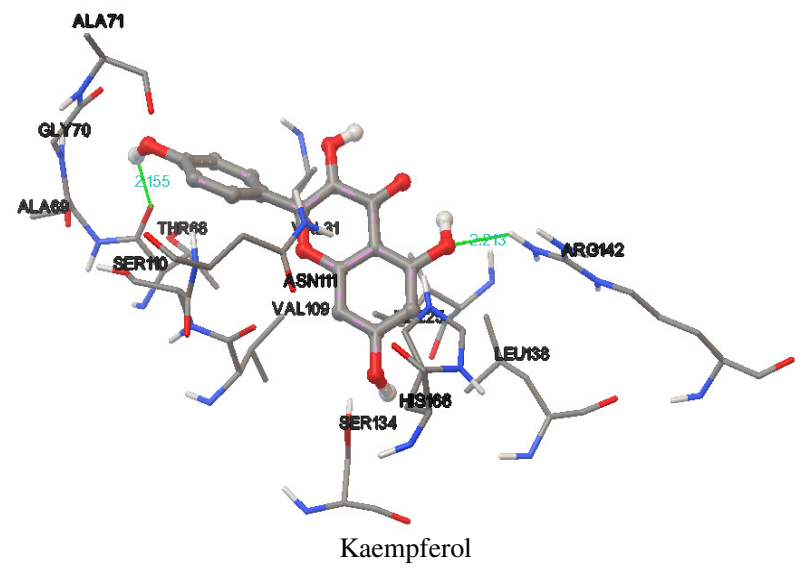

Figure 3. B4DJI1 protein-ligand interactions 


\section{Conclusion}

Model building and molecular docking study were used to attain details of interaction between protein and ligands-quercetin, naringenin, kaempferol, hesperetinand leucocyanidin. The validation of results by PROCHECK program indicated that the generated models are reliable and are analogous with the already established structures of other gingival proteins. The docking studies showed many similar residues binding with the quercetin, naringenin, kaempferol, hesperetinand leucocyanidin ligands into the active site of gingival (B4DJI1) protein. Asn111, Ala71 showed prominent interactions with four flavonoids except kaempferol. The detailed 3D structure, interaction information and the key residues identified are helpful for guiding the site-directed mutagenesis investigation.

\section{References}

1. Demmer R T and Papapanou P N, Periodontol, 2000, 2010(53), 28-44; DOI:10.1111/j.1600-0757.2009.00326.x

2. Tonetti M S, Aiuto D F, Nibali L, Donald A, Storry C, Parkar M, Suvan J, Hingorani A D and Vallance P, Deanfield $J$ Engl $J$ Med., 2007, 356, 911-920; DOI:10.1056/NEJMoa063186

3. Lalla E and Papapanou P N, Nat Rev Endocrinol., 2011, 7(12), 738-748; DOI:10.1038/nrendo.2011.106

4. Bostanci N, Ramberg P, Wahlander A, Grossman J, Jonsson D, Barnes V M and Papapanou P N, J Proteome Res., 2013, 12(2), 657-678; DOI:10.1021/pr300761e

5. Manach C, Scalbert A, Morand C, Remesy C and Jimenez L, Am J Clin Nutr., 2004, 79(5), 727-747.

6. Williams C A and Grayer R J, Nat Prod Rep., 2004, 21(4), 539-573; DOI:10.1039/B311404J

7. Cushnie $\mathrm{T}$ P and Lamb A J, Int J Antimicrob Agents, 2005, 26, 343-356; DOI:10.1016/j.ijantimicag.2005.09.002

8. Eswar N, Webb B, Marti-Renom M A, Madhusudhan M S, Eramian D, Shen M Y, Pieper U and Sali A, Curr Protoc Bioinformatics, 2006, Chapter 5: Unit 5.6.; DOI:10.1002/0471250953.bi0506s15

9. Gunda S K, AdiMahalakshmi M and Mahmood S, Int J Res Pharma Biomed Sci., 2013, 4(2), 647-655.

10. Harinda R and Nikolai P, PLoS One, 2014, 9, 1-9.

11. Laskowski R A, MacArthur M W, Moss D S and Thornton J M, J Appl Cryst., 1993, 26(2), 283-291; DOI:10.1107/S0021889892009944

12. Gunda S K, Akula L K, Shaik S, Bandi S and Shaik M, Int J Phar Sci Res., 2015, 6, 2019-2027.

13. Ranjithreddy P, Jaheer M, Reshma P and Shravan Kumar G, Int J Pharm., 2015, 5, 867-874.

14. Wass M N, Kelley L A and Sternberg M J, NAR, 2010, 38, W469-73. 